\title{
Estrategias didácticas para la enseñanza de inglés como lengua extranjera (EFL) dirigidas a estudiantes con trastorno por déficit de atención e hiperactividad (TDAH)
}

\author{
Strategies of teaching English as a Foreign Language \\ towards Attention Deficit Hyperactivity Disorder Students \\ (ADHD)
}

\author{
Gabriela De La Cruz ${ }^{1}$ \\ Magdalena Inés Ullauri Moreno ${ }^{2}$ \\ Jenny Freire ${ }^{3}$
}

\begin{abstract}
Citation/ Para citar este Artículo: De La Cruz, G., Ullauri-Moreno, M. I. y Freire, F. (2020). Estrategias didácticas para la enseñanza de inglés como lengua extranjera (EFL) dirigidas a estudiantes con trastorno por déficit de atención e hiperactividad (TDAH). Colomb. Appl. Linguistic. J., 22(2), pp. 169-182.
\end{abstract}

Received: 3-Apr.-2020 / Accepted: 30-Oct.-2020

DOI: https://doi.org/10.14483/22487085.16118

\section{Resumen}

La adquisición de un idioma es un proceso complejo, y lo es aún más para estudiantes con Trastorno por Déficit de Atención e Hiperactividad (TDAH), este trabajo propone la detección de estrategias metodológicas para la enseñanza de inglés como lengua extranjera (EFL) a un joven de 14 años con TDAH. El análisis del tema es importante puesto que existe muy poca literatura que lo aborda desde el punto de vista de la Investigación Acción Participativa (IAP) y el estudio de caso. Se entrevista a la maestra de inglés y a la psicóloga de la institución con el fin de conocer en profundidad el contexto y caracterización del estudiante. Posteriormente, durante seis sesiones se aplican estrategias didácticas como aprender sin miedo, organizar el tiempo, visualizar el trabajo, aprender jugando y evaluación de los aprendizajes las cuales influyen positivamente en la enseñanza del inglés a un estudiante con TDAH. Las fichas de observación demuestran que existen cambios en el comportamiento del estudiante después de la intervención, tales como: incremento de la atención, menos interrupciones en clase y mayor autocontrol. Los resultados permiten concluir que la aplicación de estas estrategias ajustadas al caso de estudio promueve la creatividad y la proactividad de un estudiante con TDAH.

Palabras clave: déficit de atención e hiperactividad, estrategias didácticas, estudio de caso, inglés como lengua extranjera

1 Docente de Lengua Extranjera, Universidad Nacional de Chimborazo, Ecuador. ORCID (D): https://orcid.org/0000-0002-2537-9219. gmdelacruz@unach.edu.ec

2 Docente de Lengua Extranjera, Universidad Nacional de Chimborazo, Ecuador. ORCID (D): https://orcid.org/0000-0001-5185-8566. mullauri@unach.edu.ec

3 Docente de Lengua Extranjera, Universidad Nacional de Chimborazo, Ecuador. ORCID (D): https://orcid.org/0000-0003-0065-7099. jafreire@unach.edu.ec 


\begin{abstract}
The acquisition of a language is a complex process and it is even more for students with ADHD, this work proposes the detection of methodological strategies to teach English as a foreign language (EFL) to a 14-year-old boy who has a disorder of attention deficit hyperactivity disorder (ADHD). The analysis of the topic is important since there is very little literature that addresses it using a case study. The research is qualitative and heuristic, because it aims to discover an appropriate methodology for teaching English to a young person with ADHD. The English teacher and the psychologist of the educational institution were interviewed to obtain a deep understanding of the student's context. Subsequently, various English teaching-learning methodologies were applied during 6 sessions. The observation sheets show that there are changes in the student's behavior after the intervention, such as: less interruption in class, greater self-control and less sudden movements. The results allow us to conclude that the application of methodological strategies for teaching English that promote creativity and proactivity are beneficial for a student with ADHD.
\end{abstract}

Keywords: deficit hyperactivity disorder students, ADHD, teaching strategies, case study, English as a foreign language, EFL

\section{Introducción}

A pesar de que el número de personas diagnosticadas con el Trastorno por Déficit de Atención e Hiperactividad (TDAH) no está precisado en la actualidad, se sabe que mundialmente alrededor del $5 \%$ de infantes padece TDAH y que este afecta en su mayoría a los varones en una relación de 2:1 (Asociación Americana de Psiquiatría -DSM-V, 2013; Llanos et ál., 2019).

En Estados Unidos existe una tasa entre el 3\% y el $7 \%$ de personas con TDAH; se sabe que fuera de Estados Unidos los índices son menores, lo cual se debe seguramente a la falta de diagnóstico o a diferentes metodologías de diagnóstico aplicadas (Thabet, et ál., 2010). Por su parte, según Rosero (2017), en el Ecuador existen 7918 chicos reportados en el Ministerio de Educación con TDAH en establecimientos públicos.
De las cifras anteriormente expuestas se desprende la necesidad de abordar la situación de los niños y jóvenes que tienen TDAH como un asunto de interés para las instituciones de educación básica, puesto que este síndrome influye de manera directa en el proceso de aprendizaje de los estudiantes que lo padecen y que son parte del sistema de educación preescolar, primaria y secundaria.

Según Millichap (2009) "el déficit de atención y desorden de hiperactividad es un síndrome neurológico y biológico comúnmente encontrado en niños y adolescentes, y a veces en adultos" (p.vii). El TDAH comprende los patrones de conductas de desatención, hiperactividad e impulsividad; asimismo, Millichap (2009) concibe al TDAH como "un síndrome neurológico más no una enfermedad" (p.vii). Se lo asocia comúnmente con problemas de aprendizaje puesto que las personas que sufren este desorden no llegan a obtener el nivel académico que tienen las personas de similar edad. Millichap (2009) manifiesta que por lo general el TDAH es causado por razones genéticas y medioambientales.

Para Hohman, et ál. (citado en Millichap, 2009) los niños con TDAH difieren en sus características, sin embargo, tienen en común el ser muy activos; adicionalmente, los mismos autores mencionan que las causas de este síndrome se relacionan con un daño en el cerebro. En el protocolo estipulado en el Manual de diagnóstico y estadístico de desórdenes mentales, edición $\mathrm{V}$ (DSM-V), se diferencian tres tipos de presentaciones del TDAH que son: el inatento, el hiperactivo-impulsivo y el combinado (DSM-V, 2013).

El diagnóstico se lo debe realizar por un equipo de especialistas a través de estudios: neurológicos, entrevistas personales y entrevistas con los padres de familia; como parte del protocolo de diagnóstico se debe constatar que el comportamiento siga un mismo patrón durante seis meses (Chamba, 2020).

Hidalgo, et ál. (2014) sugieren que las manifestaciones de este síndrome suelen aparecer antes de los 12 años, por lo que no solamente los médicos deben comprometerse en su diagnóstico sino también educadores y psicopedagogos. 
Los autores señalan que el Pediatra de Atención Primaria (PAP) es uno de los especialistas más importantes para tratar a los pacientes con TDAH, debido al conocimiento del niño y su familia desde el nacimiento, así como por la accesibilidad y el vínculo que tiene con el paciente hasta la adolescencia. De este modo, el diagnóstico y el tratamiento deben darse lo antes posible.

Asimismo, Clares-Almagro (2013) manifiesta que el diagnóstico se lo debe hacer de la manera más responsable posible involucrando a especialistas en el tema, padres de familia y el entorno más cercano del sujeto; para ello es recomendable que se tome un tiempo adecuado antes del diagnóstico final. Esto puede llevar meses de observación del comportamiento y desenvolvimiento del individuo en un contexto social.

El protocolo a seguir después de que una persona haya sido diagnosticada con el síndrome se basa generalmente en adaptaciones curriculares, suscripción de medicamentos, supervisión del comportamiento y consejería familiar. Asimismo, se aconsejan terapias alternativas como dietas, terapias visuales y auditivas (Millichap, 2009).

Como se señaló anteriormente, el TDAH es reconocido como un problema de aprendizaje, siendo de interés especial para este estudio el determinar los problemas de aprendizaje que un individuo que padece de TDAH, con el fin de detectar las estrategias de enseñanza-aprendizaje de un segundo idioma más eficientes y correspondientes a las características del objeto de estudio.

Para Brown (2000), el aprendizaje de un segundo idioma es largo y complejo; es necesario de un compromiso total por parte del estudiante; esto es un compromiso físico, intelectual y emocional. El autor señala además que del nivel de compromiso que el estudiante tenga, depende el éxito para el aprendizaje de una nueva lengua, una nueva cultura, una nueva manera de pensar, de sentir y de actuar. En este contexto, Brown (2000) manifiesta que es muy difícil que las personas logren alcanzar fluidez de una segunda lengua dentro de un aula de clases por lo cual, para facilitar adecuadamente el aprendizaje, Brown (2000) considera que el docente debe relacionar eficazmente muchas variables de aprendizaje, varias de ellas tienen que ver con el proceso de aprendizaje en sí.

Para Lenneber (1975), desde que los niños llegan a la pubertad es más difícil el aprendizaje de un segundo idioma, lo cual es confirmado por Piaget (1961) quien afirma que a partir de los doce años el cerebro está menos habilitado para el aprendizaje de un segundo idioma. De este modo, la adquisición de un segundo idioma para una persona de 14 años con TDAH constituye un proceso mucho más complejo de lo que usualmente es, lo cual influye directamente en el proceso de enseñanza aprendizaje del inglés como lengua extranjera.

Según Ponte (2018), los alumnos con necesidades educativas especiales necesitan una atención educativa diferente a la ordinaria. Con las llamadas adaptaciones curriculares se adaptan las necesidades de los estudiantes con TDAH y se garantiza la inclusión en la escolarización (Fernández, 2017).

Por su parte, Clares Almagro (2013) manifiesta que para enseñar a una persona con TDAH, se debe tener en cuenta la forma especial en la que estos construyen conexiones entre las diferentes partes de información y de este modo llegar al conocimiento. El mismo autor sugiere que se debe entender a la memoria como un archivo grande, un espacio infinito dentro del cual existen cajones en los que se guarda la información; lo que diferencia la forma de aprendizaje entre un individuo y otro es la habilidad con la que se rescata la información necesaria del cajón apropiado.

Siguiendo la explicación de los cajones de memoria, es muy importante entender que para una persona con TDAH es muy difícil asimilar la globalidad del mensaje si una pieza de información falta en su cerebro. Clares-Almagro (2013) señala como ejemplo que si a un niño con TDAH se le encomienda traer una naranja de la cocina y este conoce el lugar exacto en el que está la naranja, el niño con seguridad llevará la naranja solicitada; en cambio si el niño no conoce el lugar exacto en el que está la naranja en la cocina, permanecerá unos minutos jugando entretenido con cualquier otro objeto y luego regresará sin la naranja. 
El análisis de este episodio es muy importante para un profesor de inglés al ser el aprendizaje de un idioma algo muy complejo, puesto que se requiere entrelazar correctamente muchas variables. De esta manera, para lograr que el niño con TDAH aprenda inglés se requiere dotar al estudiante de toda la información necesaria para que su cerebro realice todas las conexiones necesarias previas al conocimiento.

Por su parte, Guerrero (2016) manifiesta que se deben seguir medidas estándar con el objetivo de que el alumno con TDAH se adapte al aula de clases; se recomiendan descansos cerebrales frecuentes, entender y respetar las necesidades de movimiento del alumno y ubicación adecuada del alumno en la clase asignándole un compañero que le ayude.

De esta manera, esta investigación parte de la reflexión de interrogantes como: ¿̇de qué manera debe ser el proceso de enseñanza-aprendizaje dirigido a un estudiante con TDAH?, cqué tipo de estrategias, técnicas y procedimientos se deben utilizar para que el aprendizaje sea óptimo para el estudiante con TDAH?, cंcuál es el punto de equilibrio entre lo cognitivo, afectivo y dominios físicos para el aprendizaje exitoso de un lenguaje?, cen qué casos se debe aplicar adaptaciones curriculares? y ¿de qué manera hacerlas? Las respuestas a estas interrogantes contribuyen a mejorar el proceso metodológico de la enseñanza del idioma inglés a individuos con TDAH.

En este sentido, la presente investigación muestra un estudio empírico con base teórica el cual tiene como propósito fundamental la detección de estrategias didácticas y métodos para la enseñanza de inglés como lengua extranjera a través de la determinación de los efectos académicosconductuales que estas estrategias causan en un joven de 14 , quien ha sido diagnosticado con TDAH, el mismo que es estudiante de una Unidad Educativa pública del Ecuador. Las preguntas de investigación del estudio giran en torno a cCuál es el contexto y qué características específicas presenta un estudiante que posee TDAH? y cंcuáles son las estrategias didácticas y metodologías de enseñanza de inglés como lengua extranjera acordes a un joven de 14 años que ha sido diagnosticado con TDAH?

\section{Marco teórico}

En 1902 el pediatra inglés Still (en Sabari, et ál., 2016) proporcionó la primera descripción de lo que hoy se conoce como TDAH, reconociéndolo como la presencia de conductas impulsivas y agresivas, falta de atención y problemas conductuales. Los niños con estas características eran considerados como niños "distintos", incontrolables o problemáticos. Posteriormente en 1845, Hoffman (en Sabari et al., 2016), describió por primera vez las características conductuales de algunos menores excesivamente inquietos y distraídos y dio origen al término "hiperactividad". Inicialmente, debido a que varios autores encontraron síntomas similares en niños que habían sufrido daño cerebral, se adscribió la hiperactividad a una alteración neurológica. Durante los años 30 y 40 se manejó el término de "disfunción cerebral mínima".

De acuerdo con la Asociación Americana de Psiquiatría (2013), en los años 70 se empezó a concebir al TDAH como una reacción hipercinética, que se evidencian cuando los niños menores de 5 años son inquietos y les cuesta mantener la atención (Consenso de la Sociedad Española de Psiquiatría, 2011). Para la Sociedad Española de Psiquiatría (2011) estos síntomas se vuelven un problema cuando sus manifestaciones son exageradas en relación con los otros niños y cuando estas afectan a su normal desarrollo escolar y social. Coincidiendo con lo anterior, a finales de los años 70 la novena revisión de la Clasificación Internacional de las Enfermedades CIE-9 (en Sabari, et ál., 2016) lo concibe de igual manera como un síndrome hipercinético.

En el año 1980 se publicó la tercera edición del Manual diagnóstico y estadístico de los trastornos mentales III (DSM-III), denominándolo como "trastorno por déficit de la atención". Para el año 1987 se introdujeron varias modificaciones al DSM-III y se cambió el nombre al de "trastorno por déficit de atención con hiperactividad" (Sabari, et ál., 2016, p. 558).

Existen varias clasificaciones del TDAH, sin embargo, en la revisión del DSM IV-TR (1994-2000), 
se consideran tres subtipos: inatento, hiperactivoimpulsivo y combinado. Para Mclnnes, et ál. (2003) las deficiencias del lenguaje oral también están asociadas al TDAH. Asimismo, Ygual (2012) manifiesta que los niños con TDAH presentan dificultades en el desarrollo de las habilidades metafonológicas (habilidades que implican el manejo consciente de los segmentos sonoros que integran las palabras) y no en las habilidades fonológicas, por tal razón se pueden encontrar niños que, aunque hablen correctamente, tengan problemas metafonológicos.

En la Tabla 1 se aprecia la tipología propuesta por Millichap (2009) por ser más explícita.

Con base en lo anteriormente señalado es recomendable tomar en cuenta las estrategias y técnicas de enseñanza-aprendizaje, presentes en la Tabla 2, dirigidas a estudiantes con TDAH.

\section{Metodología}

\section{Tipo de investigación}

Esta investigación tiene enfoque cualitativo y al ser un estudio de caso es descriptiva. Sobre todo, este trabajo es heurístico puesto que se pretende descubrir una metodología adecuada para la enseñanza de inglés que permita incrementar la atención de un estudiante diagnosticado con TDAH.

\section{Contexto de la investigación}

Información preliminar sobre el caso de estudio muestra que se trata de un estudiante de sexo masculino de 14 años proveniente de una familia de clase media, es el último hijo de cuatro hermanos. Sus padres poseen su propio negocio y debido al número de tareas diarias que estos realizan, el

Tabla 1. Tipos de Trastorno por Déficit de Atención e Hiperactividad TDAH.

\begin{tabular}{|c|c|c|}
\hline No. & Nombre & Criterios de detección \\
\hline 1 & Inatención sin hiperactividad & $\begin{array}{l}\text { 1. Comete errores descuidados } \\
\text { 2. No puede mantener la atención } \\
\text { 3. No escucha cuando se le habla } \\
\text { 4. No puede terminar las tareas } \\
\text { 5. Parece desorganizado } \\
\text { 6. Se distrae fácilmente }\end{array}$ \\
\hline 2 & Hiperactividad impulsiva & $\begin{array}{l}\text { 1. Nervioso } \\
\text { 2. Deja el asiento en el aula o en la mesa } \\
\text { 3. Siempre "en movimiento" } \\
\text { 4. Habla mucho }\end{array}$ \\
\hline 3 & $\begin{array}{l}\text { Inatención e hiperactividad } \\
\text { impulsiva }\end{array}$ & $\begin{array}{l}\text { Generalmente se aplica a los niños y adolescentes cuyos síntomas han disminuido } \\
\text { con la edad o el tratamiento y ya no se suman al número requerido para el } \\
\text { diagnóstico }\end{array}$ \\
\hline 4 & Trastorno de oposición desafiante & $\begin{array}{ll}\text { 1. } & \text { Pierde los estribos con frecuencia } \\
\text { 2. } & \text { Discute con los adultos } \\
\text { 3. Se niega a hacer las tareas domésticas } \\
\text { 4. Molesta a otras personas } \\
\text { 5. Jura con frecuencia }\end{array}$ \\
\hline 5 & Desorden de conducta & $\begin{array}{ll}\text { 1. } & \text { Miente } \\
\text { 2. } & \text { Juega ausente } \\
\text { 3. } & \text { Destruyó la propiedad de otros }\end{array}$ \\
\hline 6 & Desorden de ánimo & $\begin{array}{l}\text { 1. Aumento de la autoestima } \\
\text { 2. Insomnio } \\
\text { 3. Hablar en exceso }\end{array}$ \\
\hline 7 & Desorden de ansiedad. & $\begin{array}{l}\text { Los trastornos de ansiedad-pánico se caracterizan por falta de aliento, mareos, } \\
\text { frecuencia cardíaca rápida, temblores, sudoración y asfixia }\end{array}$ \\
\hline
\end{tabular}

Fuente: Millichap, (2009). 
Tabla 2. Estrategias de enseñanza-aprendizaje para estudiantes con TDAH.

\begin{tabular}{|c|c|c|c|}
\hline No. & Estrategia & Objetivo & Técnicas de aplicación \\
\hline 1 & $\begin{array}{l}\text { Aprender sin } \\
\text { miedo }\end{array}$ & $\begin{array}{l}\text { Dotar al niño con TDAH } \\
\text { de seguridad durante el } \\
\text { proceso de enseñanza- } \\
\text { aprendizaje }\end{array}$ & $\begin{array}{l}\text { Colgar el horario de clases en un sitio visible y también las normas } \\
\text { de la clase. (Clares-Almagro, 2013) } \\
\text { Para adaptar esta premisa en las clases de inglés, se puede mostrar } \\
\text { una tabla de contenidos para que el niño sepa qué tema está } \\
\text { estudiando y qué tema va después }\end{array}$ \\
\hline 2 & $\begin{array}{l}\text { Organizar el } \\
\text { tiempo }\end{array}$ & $\begin{array}{l}\text { Ayudar al niño con TDAH } \\
\text { a manejar correctamente } \\
\text { el tiempo según las tareas } \\
\text { encomendadas. }\end{array}$ & $\begin{array}{l}\text { Clares-Almagro (2013), recomienda que exista un reloj visible y que } \\
\text { se distribuyan las tareas de acuerdo con el tiempo } \\
\text { Para una clase de inglés se deberían dividir los temas y subtemas } \\
\text { en tiempos, así como las actividades a realizar por cada subtema. } \\
\text { Esta información debe estar en un lugar visible para el niño }\end{array}$ \\
\hline 3 & Visualizar el trabajo & $\begin{array}{l}\text { Tomar ventaja de la buena } \\
\text { capacidad visual de los } \\
\text { niños con TDAH }\end{array}$ & $\begin{array}{l}\text { Las tareas, así como las clases deben ser presentadas de manera } \\
\text { atractiva con colores, dibujos, interactivas etc. (Clares-Almagro, } \\
\text { 2013) } \\
\text { Una tarea atractiva de inglés puede ser que el niño ilustre (con un } \\
\text { dibujo) una conversación inicial entre dos amigos (el objetivo será } \\
\text { que el niño aprenda los saludos en inglés) }\end{array}$ \\
\hline 4 & Aprender jugando & $\begin{array}{l}\text { Enseñar inglés de una } \\
\text { manera divertida que } \\
\text { estimule sus ganas de } \\
\text { aprender }\end{array}$ & $\begin{array}{l}\text { Como sabemos, los dos inconvenientes que hay que contrarrestar } \\
\text { son la falta de atención y la hiperactividad, por lo tanto, se } \\
\text { puede apoyar en el estilo de aprendizaje del individuo y proponer } \\
\text { juegos de forma que se pueda captar su atención, y promover su } \\
\text { necesidad de movimiento } \\
\text { Por ejemplo, para enseñarle el imperative form a un niño con un } \\
\text { estilo de aprendizaje auditivo, se puede aplicar el juego de Simon } \\
\text { says en la cual se escucha comandos y se realiza la acción }\end{array}$ \\
\hline 5 & $\begin{array}{l}\text { Evaluación de los } \\
\text { aprendizajes }\end{array}$ & $\begin{array}{l}\text { Direccionar al estudiante } \\
\text { de una manera sencilla } \\
\text { sobre qué es lo que debe } \\
\text { hacer para ser evaluado }\end{array}$ & $\begin{array}{l}\text { Las preguntas deben ser cortas y con pocos apartados. No muy } \\
\text { extensas, las preguntas deben ser claras y sencillas (Clares- } \\
\text { Almagro, 2013) } \\
\text { Para evaluar los conocimientos en inglés de un niño con TDAH se } \\
\text { puede hacer pidiéndole que haga un role-play en parejas sobre los } \\
\text { saludos en el primer día de clases, completar hojas de trabajo no } \\
\text { muy extensas que tengan en su mayoría ilustraciones, preguntas } \\
\text { cortas y que sean coloridas }\end{array}$ \\
\hline
\end{tabular}

Fuente: elaboración propia con base en Clares-Almagro, M. D. (2013).

tiempo que los padres dedican para atender las necesidades de su hijo es muy limitado. El joven inició sus actividades escolares en el año lectivo septiembre 2019-junio 2020 en una nueva Unidad Educativa. Se cambia de institución debido a que tiene tercera matrícula en octavo año en la anterior institución educativa.

El equipo especialista de la Unidad Educativa actual, en coordinación con la unidad correspondiente del Ministerio de Educación, deciden aplicar la adaptación curricular de grado II, las cuales engloban también las de grado I. Según la Guía de trabajo Adaptaciones Curriculares (2013), las de grado I tienen que ver con aspectos como espacio, recursos o materiales, infraestructura, tiempo que requiere el estudiante para realizar una determinada tarea y comunicación. Mientras que, las adaptaciones curriculares de grado II implican que las estrategias metodológicas y evaluativas sean más flexibles, abiertas, innovadoras, motivadoras $y$, sobre todo, adaptables a la individualidad de cada estudiante. 
Por ejemplo, se puede proponer: tutoría entre compañeros, grupo de apoyo entre docentes, centros de interés y proyectos. Sin embargo, hay que tomar en cuenta que independientemente del tipo de adaptación curricular que se aplique, los objetivos educativos y destrezas con criterios de desempeño son iguales para todos los estudiantes.

\section{Participantes}

Para responder a las preguntas de investigación se procede a entrevistar a dos participantes: 1) la Coordinadora del Departamento de Consejería Estudiantil-DECE de la Unidad Educativa, a fin de tratar de construir en detalle el contexto del joven diagnosticado con TDAH; y 2) la maestra de inglés del estudiante con TDAH para comprender el comportamiento del joven con TDAH mientras está expuesto a las clases de inglés. Los sujetos para entrevistar han sido seleccionados a partir de su capacidad y significatividad para generar información relevante y profunda (Yuni, et ál., 2016)

\section{Estudio de caso}

Se ha escogido el estudio de caso debido a la especificidad de las características del fenómeno a investigar y además por el propósito de este. Sobre el estudio de caso, Duff (2018) considera que "son llevados a cabo por los profesores y, según los intereses, teóricos y analíticos del fenómeno, sea este lingüístico, sociolingüístico, cognitivo, político, educativo" (p.20). La misma autora manifiesta que la información recogida, la duración y el análisis e interpretación de los resultados dependen de las preguntas centrales de investigación, así como del contexto del estudio.

Por su parte, Gall, et ál. (citado en Duff, 2018) describe al estudio de caso como "un estudio específico de las características de un fenómeno en su contexto natural desde la perspectiva de los participantes como parte del fenómeno" (p. 22). En la fase de recolección de la información se presenta la caracterización del objeto de estudio de manera detallada.

En el desarrollo del estudio de caso no se aplicó ninguna técnica de muestreo, más bien se procedió a determinar que en la Unidad Educativa existía un solo caso de un joven con TDAH. En este sentido, se entrevista a la Psicóloga Coordinadora del Departamento de Consejería Estudiantil-DECE de la Unidad Educativa y a la maestra de inglés del estudiante con el fin de caracterizar al joven con TDAH.

\section{Investigación acción}

Asimismo, este trabajo responde a los principios de investigación-acción debido a que intenta solucionar un problema real vivido por los profesores (Suárez, 2002, p. 3). Para ser más precisos, este estudio se basa en los preceptos de investigación acción participativa (IAP). Para Colmenares (2012), la IAP es una metodología que presenta características particulares que la distinguen de otras, entre ellas están la manera como se aborda el objeto de estudio, las intencionalidades, el accionar de los actores involucrados, los procedimientos que se desarrollan y los logros que se alcanzan.

En este sentido, este trabajo demanda el accionar no solo del joven, del objeto de este estudio, sino también del profesor de inglés, del psicólogo educativo, de los investigadores y de las autoridades de la Institución Educativa.

Una vez contextualizado y caracterizado el objeto de estudio, se procedió a aplicar las estrategias y técnicas propuestas en la Tabla 2 y se seleccionó metodología propias de la enseñanza del inglés como lengua extranjera que ayuden en la aplicación de estas con el fin de que el estudiante con TDAH sea más proactivo durante el aprendizaje de inglés. El proceso de aplicación se realizó de forma individual durante seis sesiones, las mismas que tuvieron un tiempo de duración de 40 minutos cada una. Cada sesión se basó en un plan de clase en donde la docente de inglés abordó la misma temática que al estudiante le corresponde aprender, según la planificación curricular del noveno año de Educación Básica.

En la sesión uno se aplicaron las estrategias aprender sin miedo, visualizar el trabajo y aprender jugando para presentar el tema imperative form. Se mostró el horario de trabajo y la tabla de contenidos 
para que el estudiante esté informado, se empezó la sesión con un juego que le motive y promueva su necesidad de movimiento y se presentó el tema a ser tratado a través de presentaciones de power point y videos donde predominan las ilustraciones y los colores.

En la sesión dos se abordó la temática clase de refuerzo del imperative form y se usó la estrategia organizar el tiempo y evaluación de los aprendizajes, en donde se le asignaron tareas cortas basadas en el aprendizaje y se le estableció tiempos para la ejecución. De la misma manera en las otras sesiones.

En la sesión tres se explicó el tema present continuous mediante la utilización de las estrategias aprender sin miedo, visualizar el trabajo aprendido y aprender jugando.

La sesión cuatro intentó reforzar el tema present continous a través del uso de las estrategias organizar el tiempo y evaluación de los aprendizajes.

La sesión cinco presentó el tema Demostrative Adjectives y se aplicó las estrategias de aprender sin miedo, visualizar el trabajo y aprender jugando. Para la clase de refuerzo del tema Demonstrative Adjectives se usó las estrategias organizar el tiempo y evaluación de los aprendizajes.

En todas las sesiones se usó una presentación de power point, videos acorde a la temática, la pizarra, material multimedia implementado sobretodo para la reproducción de audio, hojas de trabajo, flash cards, reading tasks e ilustraciones.

La docente a cargo de la aplicación de las estrategias es Master en Pedagogía de los Idiomas Nacionales y extranjeros mención enseñanza de inglés y tiene cinco años de experiencia en la enseñanza del idioma y, como se mencionó en el apartado de participantes, la docente ejecuta también el rol de investigadora de este trabajo y aplicó las estrategias descritas de una manera activa con el fin de ayudar en el aprendizaje del estudiante que padece TDAH.

El detalle de la aplicación se puede observar en la Tabla 3 la cual incluye las sesiones, el contenido desarrollado, las estrategias didácticas, la metodología, las técnicas usadas y la distribución del tiempo. Cada sesión basa su desarrollo en las estrategias aprender jugando, aprender sin miedo, visualizar el trabajo, organizar el tiempo y evaluación de los aprendizajes las mismas que son adecuadas a las necesidades académicas del joven con trastorno de déficit de atención e hiperactividad.

\section{Recolección de datos}

\section{Entrevistas}

Con el fin de dar respuesta a las dos preguntas de investigación, por un lado: ¿Cuál es el contexto y qué características específicas presenta un estudiante que posee TDAH? y por otro lado, ćcuáles son las estrategias didácticas y metodologías de enseñanza de inglés como lengua extranjera acordes a un joven de 14 años que ha sido diagnosticado con TDAH?; se procede a recoger información de dos actores principales quienes laboran diariamente con el estudiante.

De esta manera, durante la recolección de la información, vía entrevista con la psicóloga y Coordinadora del Departamento de Consejería Estudiantil-DECE de la Unidad Educativa, se obtiene la siguiente información preliminar:

\section{Extracto 1 (diciembre 12 de 2019)}

El estudiante tiene problemas decomportamiento puesto que no socializa correctamente con algunos maestros. Es un poco agresivo especialmente con sus compañeros, pero a raíz de la aplicación de las adaptaciones curriculares grado Il que se han llevado a cabo, el estudiante se ha calmado y muestra mayor interés en las diferentes asignaturas impartidas.

Cabe señalar que, de la misma manera, se está llevando a cabo una reunión tipo charla semanal entre el estudiante, el tutor y la psicóloga del colegio. De igual forma, la Unidad Educativa promueve entrevistas con los padres de familia del estudiante para el seguimiento continuo del comportamiento y desempeño académico del joven. 
Tabla 3. Sesiones y contenido desarrollado.

\begin{tabular}{|c|c|c|c|c|c|}
\hline No. & Contenido & $\begin{array}{c}\text { Estrategias } \\
\text { didácticas }\end{array}$ & Métodos usados & Técnicas & Tiempo \\
\hline \multirow{4}{*}{$\begin{array}{l}\text { Sesión } \\
\text { uno }\end{array}$} & \multirow{4}{*}{ Imperative form } & \multirow{4}{*}{$\begin{array}{l}\text { Aprender jugando } \\
\text { Aprender sin miedo } \\
\text { Visualizar el trabajo }\end{array}$} & \multirow{4}{*}{$\begin{array}{l}\text { Total Physical } \\
\text { Response } \\
\text { Communicative } \\
\text { approach } \\
\text { Audiolingual method }\end{array}$} & Game "Simon says" & 10 minutos \\
\hline & & & & Showing flash cards & 10 minutos \\
\hline & & & & $\begin{array}{l}\text { Grammar explanation Watching } \\
\text { Videos and PowerPoint } \\
\text { presentations }\end{array}$ & 15 minutos \\
\hline & & & & Repetition & 5 minutos \\
\hline \multirow{3}{*}{$\begin{array}{l}\text { Sesión } \\
\text { dos }\end{array}$} & \multirow{3}{*}{$\begin{array}{l}\text { Clase de refuerzo- } \\
\text { Imperative form }\end{array}$} & \multirow{3}{*}{$\begin{array}{l}\text { Organizar el tiempo } \\
\text { Evaluación de los } \\
\text { aprendizajes }\end{array}$} & \multirow{3}{*}{$\begin{array}{l}\text { Communicative } \\
\text { approach } \\
\text { Direct method } \\
\text { Task - based } \\
\text { learning }\end{array}$} & Game "Simon says" & 10 minutos \\
\hline & & & & Worksheet & 15 minutos \\
\hline & & & & Picture-cued tasks & 15 minutos \\
\hline \multirow{3}{*}{$\begin{array}{l}\text { Sesión } \\
\text { tres }\end{array}$} & \multirow{3}{*}{$\begin{array}{l}\text { Present } \\
\text { continuous }\end{array}$} & \multirow{3}{*}{$\begin{array}{l}\text { Aprender jugando } \\
\text { Aprender sin miedo } \\
\text { Visualizar el trabajo }\end{array}$} & \multirow{3}{*}{$\begin{array}{l}\text { Total Physical } \\
\text { Response } \\
\text { Communicative } \\
\text { approach } \\
\text { Audiolingual method } \\
\text { Direct method }\end{array}$} & Game "Matching pairs" & 10 minutos \\
\hline & & & & $\begin{array}{l}\text { Grammar explanation Watching } \\
\text { Videos and PowerPoint } \\
\text { presentations }\end{array}$ & 15 minutos \\
\hline & & & & Repetition & 15 minutos \\
\hline \multirow{3}{*}{$\begin{array}{l}\text { Sesión } \\
\text { cuatro }\end{array}$} & \multirow{3}{*}{$\begin{array}{l}\text { Clase de } \\
\text { refuerzo - Present } \\
\text { continuous }\end{array}$} & \multirow{3}{*}{$\begin{array}{l}\text { Organizar el tiempo } \\
\text { Evaluación de los } \\
\text { aprendizajes }\end{array}$} & \multirow{3}{*}{$\begin{array}{l}\text { Communicative } \\
\text { approach } \\
\text { Direct method } \\
\text { Task - based } \\
\text { learning }\end{array}$} & Worksheet & 10 minutos \\
\hline & & & & Role-play & 20 minutos \\
\hline & & & & Picture-cued tasks & 10 minutos \\
\hline \multirow{3}{*}{$\begin{array}{l}\text { Sesión } \\
\text { cinco }\end{array}$} & \multirow{3}{*}{$\begin{array}{l}\text { Demonstrative } \\
\text { Adjectives }\end{array}$} & \multirow{3}{*}{$\begin{array}{l}\text { Aprender jugando } \\
\text { Aprender sin miedo } \\
\text { Visualizar el trabajo }\end{array}$} & \multirow{3}{*}{$\begin{array}{l}\text { Total Physical } \\
\text { Response } \\
\text { Communicative } \\
\text { approach } \\
\text { Audiolingual method } \\
\text { Direct method }\end{array}$} & $\begin{array}{l}\text { Singing the song "Head shoulders } \\
\text { knees and toes" }\end{array}$ & 10 minutos \\
\hline & & & & $\begin{array}{l}\text { Grammar explanation Watching } \\
\text { Videos and PowerPoint } \\
\text { presentations }\end{array}$ & 15 minutos \\
\hline & & & & Repetition & 10 minutos \\
\hline \multirow{3}{*}{$\begin{array}{l}\text { Sesión } \\
\text { seis }\end{array}$} & \multirow{3}{*}{$\begin{array}{l}\text { Clase de refuerzo } \\
\text { - Demonstrative } \\
\text { Adjectives }\end{array}$} & \multirow{3}{*}{$\begin{array}{l}\text { Organizar el tiempo } \\
\text { Evaluación de los } \\
\text { aprendizajes }\end{array}$} & \multirow{3}{*}{$\begin{array}{l}\text { Communicative } \\
\text { approach } \\
\text { Direct method } \\
\text { Task - based } \\
\text { learning }\end{array}$} & $\begin{array}{l}\text { Singing the song "Head shoulders } \\
\text { knees and toes" }\end{array}$ & 10 minutos \\
\hline & & & & Worksheet & 15 minutos \\
\hline & & & & Illustration & 15 minutos \\
\hline
\end{tabular}

Fuente: elaboración propia.

Al preguntarle cómo es el desempeño académico del estudiante, la respuesta de la Psicóloga fue la siguiente:

\section{Extracto 2 (diciembre 12 de 2019)}

Específicamente en la asignatura de inglés, el aprendizaje del estudiante y sus calificaciones fueron estables. Esto quiere decir que sus calificaciones no fueron altas, pero tampoco bajas pues en el último parcial obtuvo $7-10 . .$. Esto se debe al trabajo mancomunado entre la docente de inglés, la Psicóloga educativa, los padres de familia y el mismo estudiante sobre la base de las adaptaciones curriculares propuestas. Asimismo, se evidencia una mejora en el desempeño académico en las otras asignaturas puesto que se observa al estudiante cada vez más motivado.

En la recolección de la información se logran identificar también las fortalezas y debilidades del estudiante con el fin de conocer cuáles serían sus 
potenciales estilos de aprendizaje, la respuesta de la psicóloga al respecto fue la siguiente:

\section{Extracto 3 (diciembre 12 de 2019)}

Entre las fortalezas están que las actividades auditivas y de producción oral son las de mayor aceptación para el estudiante, y entre las debilidades se establece que el estudiante tiene dificultades en su comportamiento lo cual afecta a las relaciones sociales con algunos de sus maestros y con varios de sus compañeros por lo que no es factible realizar trabajos en grupo.

Durante la entrevista con la maestra de inglés del estudiante, al preguntarle cuál era el desempeño académico y conductual del estudiante, la entrevistada manifiesta lo siguiente:

\section{Extracto 4 (febrero 13 de 2020)}

El señor ------ es un buen chico, no molesta, a veces se distrae, pero no es todo el tiempo. Le gusta la materia porque hace preguntas y participa en clase. Al inicio, hasta que se adapte al nuevo ambiente escolar, el estudiante conversaba mucho y no participaba en clases, ahora todo ha cambiado...debe ser también porque conozco que está medicado.

\section{Ficha de observación}

Los efectos académicos y conductuales del estudiante con TDAH, objeto de estudio de esta investigación, son recolectados mediante la utilización de la ficha de observación propuesta en Loor (2019). Esta ficha de observación fue aplicada antes de las seis sesiones de clase propuestas y después de las mismas.

La observación que realizó la maestra de inglés desde su rol como docente habitual del estudiante se visualiza en la Tabla 4. Ficha de observaciónMaestra de inglés del Estudiante.

Asimismo, con el fin de contrastar los resultados, una de las investigadoras recolectó información sobre los efectos académicos y conductuales del

Tabla 4. Ficha de observación-maestra de inglés del estudiante.

\begin{tabular}{|c|c|c|c|c|c|c|c|c|}
\hline \multirow{3}{*}{ Indicador } & \multicolumn{4}{|c|}{ Previa intervención } & \multicolumn{4}{|c|}{ Post intervención } \\
\hline & \multicolumn{4}{|c|}{ Grado de efecto } & \multicolumn{4}{|c|}{ Grado de efecto } \\
\hline & Nada 0 & Poco 1 & Bastante 2 & Mucho 3 & Nada 0 & Poco 1 & Bastante 2 & Mucho 3 \\
\hline 1. Interrumpe en clases & & $\mathrm{X}$ & & & $\mathrm{X}$ & & & \\
\hline $\begin{array}{l}\text { 2. Tiene dificultades de } \\
\text { aprendizaje en el área de inglés }\end{array}$ & & $\mathrm{X}$ & & & & $\mathrm{X}$ & & \\
\hline $\begin{array}{l}\text { 3. El ritmo en la realización de las } \\
\text { tareas es muy lento en función } \\
\text { del tiempo asignado }\end{array}$ & $\mathrm{X}$ & & & & $\mathrm{X}$ & & & \\
\hline 4. Excesiva actividad motora & & & $\mathrm{X}$ & & & $\mathrm{X}$ & & \\
\hline 5. Excesiva actividad verbal & & & $\mathrm{X}$ & & & $\mathrm{X}$ & & \\
\hline 6. Falta de autocontrol & & $\mathrm{X}$ & & & & $\mathrm{X}$ & & \\
\hline 7. Movimientos repentinos & & & $\mathrm{X}$ & & & $\mathrm{X}$ & & \\
\hline 8. No puede esperar su turno & & $\mathrm{X}$ & & & $\mathrm{X}$ & & & \\
\hline $\begin{array}{l}\text { 9. Tiene dificultades en el cambio } \\
\text { de actividades }\end{array}$ & & $\mathrm{X}$ & & & & $\mathrm{X}$ & & \\
\hline $\begin{array}{l}\text { 10. Presenta una conducta } \\
\text { agresiva }\end{array}$ & & $\mathrm{X}$ & & & & $\mathrm{X}$ & & \\
\hline Total & 0 & 6 & 6 & 0 & 0 & 7 & 0 & 0 \\
\hline Efecto & & 12 previa & a intervención & & & después & la intervenciór & \\
\hline
\end{tabular}

Fuente: elaboración propia. 
estudiante con TDAH antes y después del desarrollo de las seis sesiones. Estos resultados se evidencian en la Tabla 5, Ficha de observación-investigadora.

\section{Análisis de los resultados y discusión}

Los resultados de la observación de la docente habitual de inglés expuestos en la Tabla 4, muestran que el nivel de efecto académico y conductual del trastorno del déficit de atención e hiperactividad fue superior antes de la aplicación de la estrategia didáctica propuesta, puesto que antes de la intervención el efecto era 12 y después de la intervención el efecto fue de 7; esto quiere decir que el estudiante tuvo una mejor respuesta académica y conductual después de la intervención.

Los resultados de la observación de la docente de inglés e investigadora se evidencian en la Tabla 5, cuyos indicadores fueron establecidos de acuerdo con la tipología propuesta por Millichap (2009), en donde de igual manera se aprecia un mayor nivel del efecto académico y conductual del trastorno de déficit de atención e hiperactividad antes de la aplicación de la estrategia didáctica propuesta (efecto nivel 12) lo cual quiere decir que el estudiante reaccionó de mejor manera después de la aplicación de las estrategias didácticas (efecto nivel 5).

De esta manera, se determina que las cinco estrategias didácticas, aprender sin miedo, organizar el tiempo, visualizar el trabajo, aprender jugando y evaluación de los aprendizajes junto con las metodologías y técnicas seleccionadas, son adecuadas y dan buenos resultados frente a las necesidades académicas del joven con trastorno de déficit de atención e hiperactividad, pues demostró una mayor predisposición en el proceso de aprendizaje. De la misma forma, se evidencia que el estudiante pone mayor atención a las clases, ya que estas fueron presentadas de una manera más atractiva e interactiva, llenas de colores, dibujos y videos como lo sugiere Clares-Almagro (2013).

El ritmo de realización de las tareas es mejor, ya que las actividades que debe realizar tienen tiempos determinados, el recurso del reloj visible como lo propone Clares-Almagro (2013), le permite organizar mejor su tiempo y preocuparse

Tabla 5. Ficha de observación-investigadora.

\begin{tabular}{|c|c|c|c|c|c|c|c|c|}
\hline & \multicolumn{4}{|c|}{ Previa intervención } & \multicolumn{4}{|c|}{ Post intervención } \\
\hline \multirow[t]{2}{*}{ Indicador } & \multicolumn{4}{|c|}{ Grado de efecto } & \multicolumn{4}{|c|}{ Grado de efecto } \\
\hline & Nada 0 & Poco 1 & Bastante 2 & Mucho 3 & Nada 0 & Poco 1 & Bastante 2 & Mucho 3 \\
\hline 1. Interrumpe en clases & & & $\mathrm{X}$ & & & $\mathrm{X}$ & & \\
\hline $\begin{array}{l}2 . \quad \text { Tiene dificultades de } \\
\text { aprendizaje en el área de inglés }\end{array}$ & & $\mathrm{X}$ & & & & $\mathrm{X}$ & & \\
\hline $\begin{array}{l}\text { El ritmo en la realización } \\
\text { de las tareas es muy lento en } \\
\text { función del tiempo asignado }\end{array}$ & & & $\mathrm{X}$ & & & $\mathrm{X}$ & & \\
\hline 4. Excesiva actividad motora & & $\mathrm{X}$ & & & $\mathrm{X}$ & & & \\
\hline 5. Excesiva actividad verbal & & $\mathrm{X}$ & & & $\mathrm{X}$ & & & \\
\hline Falta de autocontrol & & $\mathrm{X}$ & & & & $\mathrm{X}$ & & \\
\hline 7. Movimientos repentinos & & $\mathrm{X}$ & & & & $\mathrm{X}$ & & \\
\hline 8. No puede esperar su turno & & $\mathrm{X}$ & & & $\mathrm{X}$ & & & \\
\hline $\begin{array}{l}\text { 9. Tiene dificultades en el } \\
\text { cambio de actividades }\end{array}$ & & $\mathrm{X}$ & & & $\mathrm{X}$ & & & \\
\hline $\begin{array}{l}\text { 10. Presenta una conducta } \\
\text { agresiva }\end{array}$ & & $\mathrm{X}$ & & & $\mathrm{X}$ & & & \\
\hline Total & & 8 & 4 & & & 5 & & \\
\hline Efecto & & 12 previa & a intervención & & & después & la intervenció & \\
\hline
\end{tabular}

Fuente: elaboración propia. 
de alcanzar el objetivo, evitándose de esta manera los vacíos mentales que usualmente se forman en el cerebro del joven con TDAH cuando una pieza de información falta (Clares-Almagro, 2013).

La excesiva actividad motora también se redujo gracias a la estrategia de aprender jugando, pues se trató de aplicar juegos que propicien el movimiento al inicio de la sesión, de tal manera que su energía disminuya y pueda realizar las otras actividades. La aplicación de esta estrategia concuerda con lo estipulado por Guerrero (2016) cuando manifiesta que se recomienda establecer descansos cerebrales frecuentes así como entender y respetar las necesidades de movimiento del alumno.

Durante las seis sesiones se procuró estandarizar el proceso de enseñanza-aprendizaje mediante la aplicación de tres estrategias (aprender jugando, aprender sin miedo, visualizar el trabajo) en la sesiones donde se introduce un nuevo tema y dos estrategias (organizar el tiempo, evaluación de los aprendizajes) en la sesiones de refuerzo y de esta manera contribuir con una mejor adaptabilidad del estudiante (Guerrero, 2016).

En la evaluación de los aprendizajes, ClaresAlmagro (2013), sugiere que las preguntas deben ser cortas y con pocos apartados, no muy extensas claras y sencillas, lo cual se ha procurado aplicar en todas las actividades de evaluación con el objetivo de que el estudiante no tenga que recurrir a un análisis mental riguroso para poder responder y completar sus respuestas.

\section{Conclusiones}

El presente estudio logró el objetivo propuesto, la detección de estrategias metodológicas para la enseñanza de inglés como lengua extranjera (EFL) a un joven de 14 años con TDAH. Asimismo, se da respuesta a las dos preguntas de investigación planteadas anteriormente; así, la pregunta uno pretendía determinar ¿Cuál es el contexto y qué características específicas presenta un estudiante que posee TDAH? Después de las entrevistas realizadas, se establece que el joven tiene 14 años, pertenece a una familia de clase media y es el último hijo de cuatro hermanos. En cuanto a sus características se determina que es un joven creativo, auditivo, le gusta expresarse verbalmente y es inquieto.

En la pregunta de investigación dos ¿Cuáles son las estrategias didácticas y metodologías de enseñanza de inglés como lengua extranjera acordes a un joven de 14 años que ha sido diagnosticado con TDAH?, se identifica que el estudiante puede mejorar su aprovechamiento y comportamiento al ser instruido mediante cinco estrategias: aprender sin miedo, organizar el tiempo, visualizar el trabajo, aprender jugando y evaluación de los aprendizajes.

Las cinco estrategias didácticas fueron aplicadas junto con la ayuda de metodologías propias de la enseñanza del inglés como direct method, audio lingual, total physical response, the communicative approach, and task-based learning, las mismas que ayudaron en el proceso y se ajustaron a los requerimientos del estudiante.

Es importante aplicar las estrategias con base en las características de cada individuo, por ello es necesario en primera instancia, detectar sus miedos, gustos, fortalezas e intereses y de esa manera proponer actividades atractivas, permitiendo siempre el movimiento y respetando su individualidad.

El manejo de los tiempos es un aspecto muy importante pues la atención se pierde con actividades muy prolongadas, por lo que se debe tomar en cuenta la utilización del reloj, mostrar la planificación de la clase y estandarizar los procesos.

Al término del estudio, los dos observantes notaron cambios en el comportamiento del estudiante antes y después de la intervención, tales como: más motivación en el aprendizaje, menos desconcentración e interrupciones y mejora en el rendimiento.

A pesar de que estos resultados no se puedan generalizar con todos los estudiantes de inglés que tengan TDAH, estos pueden ser de utilidad al menos para estudiantes con características similares al caso presentado. 
Finalmente, se puede destacar que el síndrome del TDAH no es una enfermedad sino más bien una característica individual de algunos estudiantes, que puede ser mitigada y controlada por los agentes de la sociedad que se encuentran es su entorno, docentes, padres de familia, psicólogos, compañeros de clase, amigos, etc.

\section{Referencias}

American Psychiatric Association. (2013). Diagnostic and statistical manual of mental disorders (DSM-5®). American Psychiatric Pub.

Brown, H. (2000). Principles of language learning and teaching (Vol. 4). Longman.

Clares-Almagro, M. (2013). TDAH y el aprendizaje de inglés en la escuela: Una propuesta metodológica [Tesis de pregrado]. Universidad Internacional de la Rioja.

Chamba, K. (2020). Taller de vinculación Maestría PINE. [video de https://www.youtube.com/ watch?v=QrvaKcKfewY

Colmenares, E. (2012). Investigación-acción participativa: una metodología integradora del conocimiento y la acción. Voces y Silencios. Revista Latinoamericana de Educación, 3(1), 102-115.

Duff, P. (2018). Case study research in applied linguistics. Routledge.

Fernández, I. (2017). Estudio empírico sobre la aplicación de los principios del Diseño Universal para el Aprendizaje en la enseñanza de inglés como lengua extranjera en niños con Trastorno por Déficit de Atención con Hiperactividad (TDAH). [Tesis doctoral]. Universidad de Extremadura.

Guerrero, R. (2016). Trastorno por déficit de atención con hiperactividad. Planeta.

Hidalgo, M. y Santos, L. (2014). Trastorno por déficit de atención e hiperactividad. Manifestaciones clínicas y evolución. Diagnóstico desde la evidencia científica. PediatríaIntegral, 609.

Lenneberg, E. (1975). Fundamentos biológicos del lenguaje. Alianza Editorial.

Llanos, L., García, D., González, H., y Puentes, P. (2019). Trastorno por déficit de atención e hiperactividad (TDAH) en niños escolarizados de 6 a 17 años. Pediatría Atención Primaria, 21(83), e101-e108. http://scielo.isciii.es/scielo.php?script $=$ sci arttextEpid=S1139-76322019000300004Elng =es $\underline{\varepsilon t \operatorname{lng}=\mathrm{es}}$
Loor, K. (2019). Efectos académicos y conductuales del trastorno de déficit de atención e hiperactividad en los estudiantes de segundo a séptimo año de educación general básica de la Unidad Educativa Raúl González Astudillo. [Tesis de pregrado]. Pontificia Universidad Católica del Ecuador sede Santo Domingo, Santo Domingo.

McInnes, A., Humphries, T., Hogg-Johnson, S., and Tannock, R. (2003). Listening comprehension and working memory are impaired in attentiondeficit hyperactivity disorder irrespective of language impairment. Journal of abnormal child psychology, 31(4), 427-443. https://doi. org/10.1023/A:1023895602957

Millichap, J. (2009). Attention deficit hyperactivity disorder handbook: A physician's guide to ADHD. Springer Science $\&$ Business Media.

Ministerio de Educación. (2013). Guía de trabajo Adaptaciones Curriculares para la educación especial e inclusiva. República del Ecuador. http://fesvip.edu.ec/assets/guia-adaptacionescurriculares-3-(1).pdf

Piaget, J. (1961). La formación del símbolo en el niño. Fondo de Cultura Económica.

Ponte, A. (2018). Tratamiento del Trastorno por Déficit de Atención e Hiperactividad (TDAH) en el aula de lengua extranjera de Educación Primaria. [Tesis de pregrado]. Universidad Internacional de la Rioja.

Rosero, M. (2017, mayo 1). El déficit de atención se detecta cuando el niño va a la escuela. El Comercio. https://www.elcomercio.com/tendencias/detecciondeficit-atencion-ninos-escuela.html

Sabari, A., Naranjo, M., Torres, M. y García, C. (2016). Trastorno por déficit de atención e hiperactividad: algunas consideraciones sobre su etiopatogenia y tratamiento. Medisan, 20(4).

Suárez P. (2002). Algunas reflexiones sobre la investigación acción colaboradora de la educación. Revista Electrónica de Enseñanza de las Ciencias, 1(1). http://disde.minedu.gob.pe/xmlui/ bitstream/handle/123456789/1835/Algunas\%20 reflexiones\%20sobre\%20la\%20investigacionaccion $\% 20$ colaboradora $\% 20$ de $\% 201$ a $\% 20$ educacion.pdf?sequence $=1$

Sociedad Española de Psiquiatría. (2011). TDAH y Trastorno Hipercinético. Madrid, España: Leaflets Department. $\quad$ http://www.sepsiq.org/file/Royal/5TDAH.pdf

Thabet, A., Al Ghamdi, H., Abdulla, T., Elhelou, M. y Vostanis, P. (2010). Attention deficit-hyperactivity symptoms among Palestinian children. EMHJ Eastern Mediterranean Health Journal, 16 (5)), 505510. 
Ygual, A. (2012). El procesamiento de habla en niños con trastorno por déficit de atención con hiperactividad: Intervención. Universitat de València. Estudi General. Departamento de Psicología Evolutiva y de la Educación. Trabajo presentado en la XIII Curso Internacional de Actualización en Neuropediatría y Neuropsicología Infantil.
Yuni, J. y Urbano C. (2016). Técnicas para investigar: recursos metodológicos para la preparación de proyectos de investigación. Brujas.

\section{(c) (i) $\circledast 9$

